\title{
Relativistic Gravitational Field and Invalidity of Singularity
}

\author{
Bruno Prabagaran Mathalaisamy \\ HCL Technologies, Chennai, India \\ Email: brunoprabagaran.m@hcl.com
}

How to cite this paper: Mathalaisamy, B.P. (2021) Relativistic Gravitational Field and Invalidity of Singularity. Journal of High Energy Physics, Gravitation and Cosmology, 7, 1102-1106.

https://doi.org/10.4236/jhepgc.2021.73065

Received: April 28, 2021

Accepted: July 5, 2021

Published: July 8, 2021

Copyright (c) 2021 by author(s) and Scientific Research Publishing Inc. This work is licensed under the Creative Commons Attribution-NonCommercial International License (CC BY-NC 4.0). http://creativecommons.org/licenses/by-nc/4.0/

\begin{abstract}
A century of successful experiments on general relativity was unable to convince substantial scholars on the presence of "gravitational singularity". Physically questionable outcomes of general relativity are due to the deployment of non-relativistic Newtonian gravitation. However, the nonrelativistic classical gravitational field equation can be utilized for the weak field with negligible error. The connection between inertia and mass is distinct for classical and relativistic mechanics. As per the equivalence principle, the connection between energy and spacetime should be identical for special and general relativity; the relativistic approach on the gravitational field will eliminate "gravitational singularity". This theory aligned well with relativity and improved sturdiness to general relativity. The impacts of this model on general relativity experimental results are insignificant.
\end{abstract}

\section{Keywords}

Relativity, Gravitation, Singularity, Black Hole

\section{Introduction}

We use inertia or gravity as a tool to identify the mass of matter. However, application of Newtonian physics to detect the mass of a high-speed particle, by its kinetic energy, will result in the wrong measurement. The resistance exerted by spacetime on to the matter does not agree with the interpretation of classical mechanics. Therefore, the established classical link between mass and inertia is profoundly inaccurate. Einstein's theory of special relativity (SR) is well established, and it asserts that infinite resistance is exerted on matter when it approaches the speed of light [1]. With the help of relativity, the inevitability of the Lorentz factor (LF) in the kinetic energy of a high-speed particle is experimentally verified [2]. Therefore, the spacetime fabric can produce infinite resistance 
as per SR.

Like inertia, the classical connection between energy and gravitational field should be modified with relativistic mechanics, retaining the underlying hypothesis of general relativity (GR). As being reasoned, we can arrive to the following assumptions:

- Relativistic mass or momentum effect should be due to the resistance produced by the spacetime fabric [3].

- Regarding gravity, the resistance offered by spacetime fabric to curve must be associated with the gravitational field producing energy/mass.

\section{Applying Relativistic Approach on Gravitational Field}

In GR, the influence of the gravitational field on time and space was addressed; however, the Newtonian mechanics were used in approximation to define the spacetime metrics at any given point in the space [4]. Relativistic energy incorporated in the energy-momentum-stress tensor only helps to determine the whole energy in the system. Hence, the relativistic approach was not deployed to connect mass and the respective spacetime curvature, and that caused the GR "Schwarzschild radius" to be equivalent to classical mechanics [5]. In this article, we will focus only on invalidating the singularity outcome by modifying the classical gravitational field equation.

Lorentz factor is $(\gamma)$,

$$
\frac{1}{\sqrt{1-\frac{v^{2}}{c^{2}}}}
$$

where $v$ is the velocity of the freefalling object and $c$ is the velocity of light.

Gravitational potential energy $(E)$, as per classical mechanics equals to:

$$
E=\left(\frac{G M m}{r}\right)
$$

where $G$ is the gravitational constant, $M$ is the gravitational mass, $m$ is the mass of freefalling object, and $r$ is the distance from the center of mass.

Energy required to accelerate the object to velocity $V$, as per the relativistic mechanics equals to:

$$
\frac{E}{\gamma}=\frac{1}{2} m v^{2}
$$

As examined, if inertial/kinetic energy is subject to resistance from spacetime, LF must be considered to link energy/mass and its gravitational spacetime curvature. Here $v^{2}$ primarily embodies the spacetime curvature and its outcome is velocity of the freefalling object.

Inserting Equation (1) in Equation (2), we obtain,

$$
\left(\frac{G M m}{r}\right) \frac{1}{\gamma}=\frac{1}{2} m v^{2}
$$




$$
\begin{gathered}
\left(\frac{2 G M}{r}\right) \frac{1}{\gamma}=v^{2} \\
\left(\frac{2 G M}{c r}\right)^{2}\left(c^{2}-v^{2}\right)=v^{4}, \frac{2 G M}{c r}=\kappa \\
v^{2}=\frac{1}{2} \kappa\left\{\sqrt{\kappa^{2}+4 c^{2}}-\kappa\right\}
\end{gathered}
$$

Classical gravitational field equation:

$$
g=-\nabla \Phi=\frac{G M}{r^{2}}
$$

where $g$ is the gravitational acceleration and $\Phi$ is gravitational potential.

Replace $\frac{2 G M}{r}$ in Equation (4) with $v^{2}$ in Equation (3) to get relativistic gravitational field equation.

$$
g=-\nabla \Phi=-\frac{1}{2} \nabla v^{2}
$$

\section{Results}

With the help of the new equation, we can conclude that infinite energy/matter is required to produce the "event horizon" irrespective of the coordinate system. Compared to the classical mechanics, gravitational acceleration gradient reduces further and further near the supermassive object.

\subsection{Data within the Solar System}

Following is the comparison of current and proposed methods regarding the escape velocity of earth and sun; it illustrates the precision of this theory in a weak gravitational field.

Earth: $\left(\right.$ Mass $=5.972 \times 10^{24} \mathrm{~kg}[6]$, Radius $=6,371,000 \mathrm{~m}$ [6], Gravitational Constant $\left.=6.6743 \times 10^{-11} \mathrm{~m}^{3} \cdot \mathrm{kg}^{-1} \cdot \mathrm{s}^{-2}[7]\right)$.

Escape velocity by existing method $=11,186.3524997063 \mathrm{~m} / \mathrm{s}$

Escape velocity by proposed method $=11,186.3524958126 \mathrm{~m} / \mathrm{s}$

Sun: $\left(\right.$ Mass $=1.9885 \times 10^{30} \mathrm{~kg}[8]$, Radius $=695,700,000 \mathrm{~m}$ [8], Gravitational Constant $\left.=6.6743 \times 10^{-11} \mathrm{~m}^{3} \cdot \mathrm{kg}^{-1} \cdot \mathrm{s}^{-2}[7]\right)$.

Escape velocity by existing method $=617,688.6989 \mathrm{~m} / \mathrm{s}$.

Escape velocity by proposed method $=617,688.0433 \mathrm{~m} / \mathrm{s}$.

Based on the above comparison, we can conclude that the redshift and gravitational lensing experiments performed within the solar system will comply with the proposed changes.

\subsection{Gravitational Time Dilation}

Current gravitational time dilation equation [9],

$$
\frac{\tau}{t}=\sqrt{1-\frac{2 G M}{r c^{2}}}
$$


where $\tau$ is the time between two events for an observer close to the massive object and $t$ is the time between the events for an observer at an arbitrarily large distance from the massive object.

Replace $\frac{2 G M}{r}$ in Equation (6) with $v^{2}$ in Equation (3),

$$
\frac{\tau}{t}=\sqrt{1-\frac{v^{2}}{c^{2}}}
$$

Figure 1 shows a comparison of gravitational time dilation of the current and proposed technique for the solar mass object. In the current method, time stops at the Schwarzschild radius of $2953 \mathrm{~m}$ for the solar mass object, but in our proposed method, "zero" Schwarzschild radius is required to produce the corresponding effect on time.

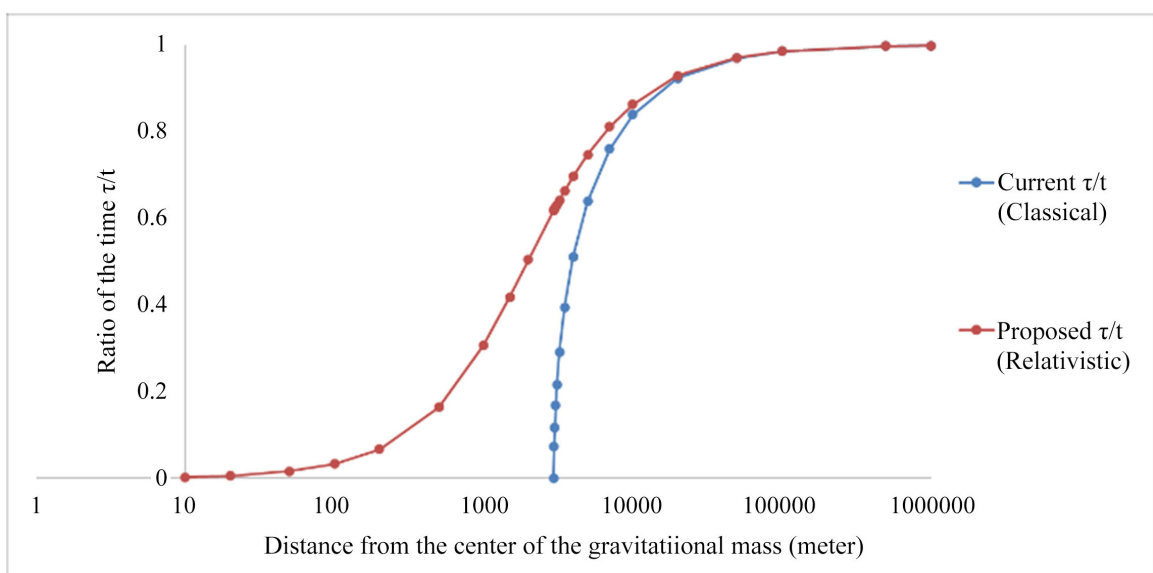

Figure 1. Comparison of gravitational time dilation.

\subsection{Influence on the Extreme Gravitational Field Experiments}

The ratio between the extreme gravitational potential of the current and proposed method in Schwarzschild precession tests performed on orbits of star S2 near the galactic center will provide the same result to the order of $10^{-3}$.

Extreme gravitational potential $(\Phi)$ of $\operatorname{Sgr} \mathrm{A}^{*}$, as per the data in study is listed below.

$$
\begin{aligned}
& \text { Mass } \approx 4.25 \times 10^{6} \mathrm{M}_{\odot}[10], \text { Radius (pericenter) } \approx 120 \mathrm{AU}[10] ; \\
& \Phi_{\text {Current method }} \approx 59,141,880,107,879 \mathrm{~J} / \mathrm{kg} ; \\
& \Phi_{\text {Proposed method }} \approx 59,122,424,383,746 \mathrm{~J} / \mathrm{kg} .
\end{aligned}
$$

Gravitational waves can be produced by supermassive objects with no event horizon; it does not prove the existence of gravitational singularity.

These experiments are examined to show the validity of the proposed theory, and that is sufficient to prove compliance with other experiments. Further profound studies are needed to be performed in the future.

\section{Conclusion}

Indeed, gravitational singularity is not supposed to exist as per relativity. There 
is no solid evidence for the existence of supper massive objects with an event horizon. The article shows why the consideration of relativity in the gravitational field is crucial, and GR became more reliable by eliminating physically objectionable outcomes (singularity; event horizon). Even though the Einstein field equation was not assessed as part of this study, we have evaluated how classical physics is directly applied in GR. It is apparent that the proposed changes will only have a negligible impact on the results of tests performed on GR. This study will open new doors for a different perspective and enhanced research.

\section{Conflicts of Interest}

The author declares no conflicts of interest regarding the publication of this paper.

\section{References}

[1] Einstein, A. (1998) On the Electrodynamics of Moving Bodies. In: Stachel, J., Ed., Einstein's Miraculous Year, Princeton University Press, USA.

[2] Luetzelschwab, J.W. (2003) Apparatus to Measure Relativistic Mass Increase. American Journal of Physics, 71, 878-884. https://doi.org/10.1119/1.1561457

[3] Taylor, E.F. and Wheeler, J.A. (1992) Spacetime Physics. Second Edition, W.H. Freeman and Company, New York, 248-249.

[4] Einstein, A. (1952) The Foundation of the General Theory of Relativity. Dover, New York.

[5] Lindner, H.H. (2012) Beyond Newton and Einstein to Flowing Space. Physics Essays, 25, 500-509. http://henrylindner.net/Writings/BeyondNewton.pdf https://doi.org/10.4006/0836-1398-25.4.500

[6] Williams, D.R. (2017) Earth Fact Sheet. NASA/Goddard Space Flight Center. https://nssdc.gsfc.nasa.gov/planetary/factsheet/earthfact.html

[7] 2018 CODATA Value: Newtonian Constant of Gravitation. The NIST Reference on Constants, Units, and Uncertainty. NIST. https://physics.nist.gov/cgi-bin/cuu/Value?bg

[8] Williams, D.R. (2013) Sun Fact Sheet. NASA Goddard Space Flight Center. https://nssdc.gsfc.nasa.gov/planetary/factsheet/sunfact.html

[9] Ryder, L. (2009) Einstein Field Equations, the Schwarzschild Solution and Experimental Tests of General Relativity. In: Introduction to General Relativity, Cambridge University Press, Cambridge, 137-179. https://doi.org/10.1017/CBO9780511809033.007

[10] Abuter, R., Amorim, A., Bauböck, M., et al. (2020) Detection of the Schwarzschild Precession in the Orbit of the Star S2 Near the Galactic Centre Massive Black Hole. $A \& A, 636$, Article No. L5. https://doi.org/10.1051/0004-6361/202037813 